University of Nebraska - Lincoln

DigitalCommons@University of Nebraska - Lincoln

August 1987

\title{
Mode anharmonicity in molecular crystals studied by piezomodulated Raman spectrosocpy
}

\author{
K.M. White \\ University of Nebraska - Lincoln \\ Craig J. Eckhardt \\ University of Nebraska - Lincoln, ceckhardt1@unl.edu
}

Follow this and additional works at: https://digitalcommons.unl.edu/chemistryeckhardt

Part of the Chemistry Commons

White, K.M. and Eckhardt, Craig J., "Mode anharmonicity in molecular crystals studied by piezomodulated Raman spectrosocpy " (1987). Craig J. Eckhardt Publications. 27.

https://digitalcommons.unl.edu/chemistryeckhardt/27

This Article is brought to you for free and open access by the Published Research - Department of Chemistry at DigitalCommons@University of Nebraska - Lincoln. It has been accepted for inclusion in Craig J. Eckhardt Publications by an authorized administrator of DigitalCommons@University of Nebraska - Lincoln. 


\title{
Mode Anharmonicity in Molecular Crystals Studied by Piezomodulated Raman Spectroscopy
}

\author{
K. M. White and C. J. Eckhardt ${ }^{(a)}$ \\ Department of Chemistry, University of Nebraska-Lincoln, Lincoln, Nebraska 68588
}

(Received 23 March 1987)

\begin{abstract}
Piezomodulated Raman scattering is used to determine the extent of anharmonicity, the phononphonon coupling, and the presence of modes involved in the phase transition of 1,2,4,5-tetrabromobenzene. The experiments support earlier theoretical predictions and demonstrate that piezomodulated Raman spectroscopy is an important new approach to the experimental study of lattice dynamics.
\end{abstract}

PACS numbers: $63.20 . \mathrm{Ry}, 63.20 . \mathrm{Hp}, 64.70 . \mathrm{Kb}, 78.30 . \mathrm{Jw}$

Lattice anharmonicity, while extensively investigated for crystals displaying significant long-range interactions such as ionics, has only recently been examined for molecular crystals. These systems have been largely ignored although they effectively allow study of the contribution of short-range interactions to the anharmonicity. They are of additional interest because they provide favorable opportunities for investigation of the coupling of internal and external modes. From the standpoint of intermolecular interactions, the molecular crystal has provided the most widely used parametrizations for intermolecular potentials, and has yielded the most varied models for the resulting interactions. While the role of anharmonicity is of consequence to all of these concerns, it remains a most difficult property to probe quantitatively. Of course, the importance of anharmonicity is not limited to molecular crystals, but is of concern for all lattices.

Anharmonicity is manifested by shifts of phonon frequencies from harmonic values and the finite lifetime of phonon states. Neither is easy to assess, especially for the lattice modes of molecular crystals. Determination of the frequency shifts requires overtone and combination bands which appear most infrequently in molecular crystals. Bandwidth measurements allow study of the lifetimes, although complications involving band shapes are often inhibiting.

Recently, a theoretical basis has been established for studying the anharmonicity of crystals by piezomodulated Raman spectroscopy. ${ }^{1}$ The essential idea is that the periodic stress applied to a crystal enhances phononphonon coupling which is mediated by the acoustic phonons associated with the essentially static (on the time scale of the interactions) strain field. The spectra obtained represent the difference between responses at the extremes of compression and extension of the crystal. It is necessary to formulate a renormalized Hamiltonian, which is obtained by considering only modes at the center of the Brillouin zone and partitioning them as

$$
Q(0, j)=Q_{j}^{\mathrm{el}}+Q_{j},
$$

where $Q_{j}^{\text {el }}$ is the elastic displacement of the molecules for a given strain $e_{\alpha \beta}$, and $Q_{j}$ represents the vibrations about this instantaneous equilibrium position. The effective Hamiltonian for the unit cell of volume $v$ of the deformed lattice becomes

$$
\begin{aligned}
H=H_{0}+\frac{1}{2} v \sum_{\alpha \beta \gamma \delta} & C_{\alpha \beta \gamma \delta} e_{\alpha \beta} e_{\gamma \delta} \\
& +\frac{1}{2} \sum_{j j^{\prime}}\left[\omega_{0 j}^{2} \delta_{j j^{\prime}}+\sum_{\alpha \beta} \phi_{\alpha \beta, j j^{\prime}} e_{\alpha \beta}\right] Q_{j} Q_{j^{\prime}},
\end{aligned}
$$

where $H_{0}$ is the Hamiltonian for the undeformed lattice, $C_{\alpha \beta \gamma \delta}$ is the effective elastic constant, and the coupling between the optical phonons due to strain is given by $\phi_{\alpha \beta, j j^{\prime}}$. The term on the second line represents the anharmonicity.

Definition of the electronic susceptibility in terms of the renormalized coordinates and representation of the Raman intensity by the correlation of the susceptibility lead to an expression for the intensity of the inelastically scattered light affected by the strain. For the onephonon oscillator approximation with an associated susceptibility $\chi$, the intensity is

$$
\Delta I(\omega)=-e_{\alpha \beta} \operatorname{Im}\left[\chi(\omega) \phi_{\alpha \beta} \chi(\omega)\right] .
$$

Thus, the crucial result of the analysis is that the strength of the response, which is essentially proportional to the stress derivative of the normal Raman spectrum, will depend on the amount of coupling between the modes which is induced by the strain. An ideally harmonic mode will give no piezomodulation signal since the coupling constant, $\phi_{\alpha \beta}$, would be zero. At this juncture the discussion is general, and the expression for the susceptibility is governed by the type of lattice which is being investigated.

Further, the measurement can be used to determine the anisotropy of the recently proposed stress Grüneisen parameter. $^{2}$ This is of particular usefulness since, for molecular crystals, knowledge of the stress Grüneisen parameters permits extraction of information on both the harmonic and anharmonic contributions to the intermolecular potential. ${ }^{3}$

Because both the strain and the phonon coordinate may serve as order parameters in phase transitions, piezomodulated Raman spectroscopy should serve as an 
effective probe of systems which undergo phase transitions. A preliminary study ${ }^{4}$ on anthracene crystals has shown that the response of the piezomodulated Raman spectrum in the lattice-mode region is consistent with the suggestion of the existence of a triclinic intergrowth phase. ${ }^{5}$ However, piezomodulated Raman spectroscopy has not yet been shown to present an unambiguous response as predicted by the theory. The potential importance of piezomodulated Raman spectroscopy to the study of lattice dynamics has prompted this investigation of the $\beta$ phase of 1,2,4,5-tetrabromobenzene (TBB). Study of a molecular crystal is desirable because of the clear separation of the internal and external modes. The former can be expected to be approximately harmonic and to provide a useful test of the predictions.

At atmospheric pressure, TBB exhibits a structural phase transition at $T_{c}=319.7 \quad \mathrm{~K},{ }^{6}$ where the lowtemperature $\beta$ phase transforms to the $\gamma$ phase which persists until the melting point. Both phases are monoclinic $p 2_{1} / a$, with the two molecules in the unit cells located at inversion centers. ${ }^{7}$ The unit-cell parameters and spatial dispositions of the molecules closely resemble each other for the two phases. The transition appears to be displacive ${ }^{8}$ and is destructive. ${ }^{7}$ Current values for bromine atom-atom potentials in the literature have proven unreliable for a useful lattice dynamical calculation thereby preventing a microscopic analysis of the modes involved in the phase transition. ${ }^{9}$

The Raman spectra in the lattice-mode region of the two phases differ significantly from each other. ${ }^{8,10}$ The $22-\mathrm{cm}^{-1}$ band in the $\beta$ phase decreases in intensity with temperature, decreasing to zero at $T_{c}$; the wave-number shift of this band from $300 \mathrm{~K}$ to $T_{c}$ is less than $1 \mathrm{~cm}^{-1}$. In the $\gamma$ phase a new band is seen at $28 \mathrm{~cm}^{-1}$. Such behavior suggests that these modes, although apparently not soft modes at $q=0$, are strongly anharmonic.

Modes which are identified with the molecular vibrations are expected to be relatively harmonic since they are substantially remote from the lattice modes. In TBB the Raman-active internal modes show a gap of $80 \mathrm{~cm}^{-1}$ from the external modes. In addition, the infrared spectra for TBB indicate that internal modes above 1000 $\mathrm{cm}^{-1}$ are insensitive to lattice deformation. ${ }^{11}$ The piezomodulated Raman spectra are reported here for the $22-\mathrm{cm}^{-1}$ lattice mode and the $1122-\mathrm{cm}^{-1}$ molecular mode.

The TBB was obtained from Aldrich Chemical and purified by vacuum sublimation and recrystallization. Single crystals grown from chloroform were mounted in an anvil arrangement between a lead zirconate titanate (PZT) piezoceramic bar and a steel plate. The periodic stress was obtained by driving the PZT bar with a peak voltage of $750 \mathrm{~V}$ at $200 \mathrm{~Hz}$. The uniaxial stress direction, denoted by $[110]^{*}$, was normal to $(110)$, and perpendicular to the $c$ axis of the crystal. The resulting strain of 0.001 was determined from the displacement of the PZT bar since the crystal is extremely compliant. The optical signal was synchronously detected with use of a lock-in amplifier. Temperature was controlled by blowing air warmed to the desired temperature which was monitored near the crystal. The temperature varied by less than half a degree.

The scattered light from the $5145-\AA \mathrm{Ar}^{+}$-laser exciting line was gathered in the $90^{\circ}$ scattering geometry, and was dispersed by a Spex model 1400 double monochromator. The incident light was directed along the crystal $c$ axis with polarization along the $b$ axis. The scattered light collected normal to the (110) face was polarized along $c^{\prime}$ for the lattice modes, and $c^{\prime \prime}$ for the molecular modes, where $c^{\prime}$ and $c^{\prime \prime}$ are the principal directions on ( $1 \overline{1} 0)$.

Single-crystal Raman spectra were obtained at $300 \mathrm{~K}$ with frequencies in agreement with those reported earlier. ${ }^{8,10}$ The piezomodulated Raman spectrum was measured for the $22-\mathrm{cm}^{-1}$ mode of the $\beta$ phase and is shown in Fig. 1. To clarify further the nature of the piezomodulation response, the spectrum of an internal mode at $1122 \mathrm{~cm}^{-1}$ was also obtained at $300 \mathrm{~K}$. This is also shown in Fig. 1. Although the Raman line for the molecular-ring breathing mode ${ }^{9}$ at $1122 \mathrm{~cm}^{-1}$ is itself only $10 \%$ of the intensity of the lattice mode, its piezomodulation response (essentially at the level of the noise) is, in contrast, 250 times smaller than that of the 22$\mathrm{cm}^{-1}$ mode. While not studied in detail, piezomodulated responses were obtained for other lattice modes, but they were significantly smaller than those observed for the $22-\mathrm{cm}^{-1}$ mode which is apparently much more involved with the phase transition. These observations support the theoretical analysis which predicts that the

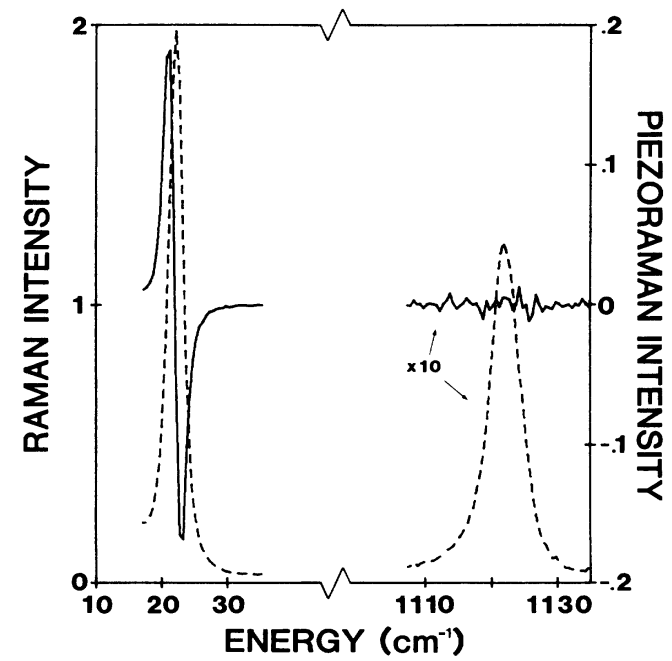

FIG. 1. Raman (dashed line) and piezomodulated Raman (solid line) spectra of the $22-\mathrm{cm}^{-1}$ lattice and $1122-\mathrm{cm}^{-1}$ intramolecular modes in TBB at $300 \mathrm{~K}$. All spectra are plotted on the same relative intensity scale in arbitrary units. 
strong signals should arise from the more anharmonic modes.

Further evidence of the sensitivity of the piezomodulated Raman spectroscopy to anharmonicity is found by obtaining the modulated spectra in the region of the 22$\mathrm{cm}^{-1}$ band for several temperatures. These are shown in Fig. 2. At $300 \mathrm{~K}$ only a single, derivativelike band is observed. However, at $308 \mathrm{~K}$ another band is seen to develop near $28 \mathrm{~cm}^{-1}$, even though there is no equivalent structure observed in the direct Raman spectrum. At approximately $310 \mathrm{~K}$ this new band is observed in the direct Raman spectrum, which is associated with a significantly increased response in the piezomodulated Raman spectrum. When the temperature is increased to $313 \mathrm{~K}$ the intensity of the $22-\mathrm{cm}^{-1}$ band is greatly diminished, as is its piezomodulated response, and the 28 $\mathrm{cm}^{-1}$ mode is now dominant in both spectra. Above $T_{c}$ the $22-\mathrm{cm}^{-1}$ band has disappeared.

Additional information is conveyed by the sense of the piezomodulated signal. Since the differential response is between the extensive minus compressive states of the crystal, the piezomodulation spectrum of the $22-\mathrm{cm}^{-1}$ band shows that the mode shifts to higher energy for compressive stress along [110]* This is consistent with the results of hydrostatic-pressure studies on molecular crystals. Because of the uniaxial nature of the experi-

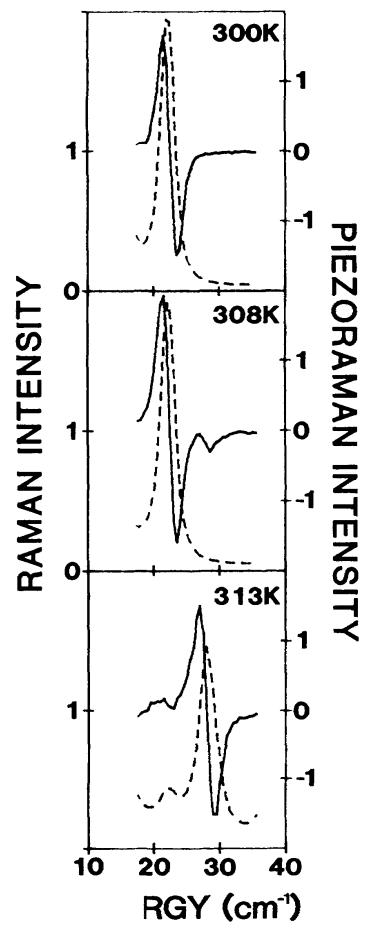

FIG. 2. Raman (dashed line) and piezomodulated Raman (solid line) spectra for the $22-\mathrm{cm}^{-1}$ mode at temperatures approaching the structural phase transition temperature. The left and right intensity scales in arbitrary units are related by a constant factor. ment, however, this may not always be expected. The piezomodulation experiment therefore provides new avenues for investigation of the microscopics of the lattice potential.

The data permit calculation of the coupling constant defined above. A theoretical fit to the Raman line at 22 $\mathrm{cm}^{-1}$ with an expression developed previously ${ }^{1}$ yields a value for the coupling parameter, $\phi_{[110]^{*}}$, of $2625 \pm 875$ $\mathrm{cm}^{-2}$. For the strain suffered by the crystal, this represents a correction of only $1 \%$ to the harmonic response (third term of the Hamiltonian above) for this mode. The frequency shift arising from the strain is $0.09 \pm 0.03 \mathrm{~cm}^{-1}$, which is consistent with that expected from calculation of the stress Grüneisen parameters for molecular crystals. ${ }^{1}$ The experimental accuracy is limited by the perfection of the samples and represents no limitation of the technique.

The lack of known elastic constants for TBB prevents further analysis of the data to obtain the stress Grüneisen parameters. However, there is no reason these could not be obtained for crystals with known compliances.

These results clearly support the prediction of the theory and demonstrate that piezomodulated Raman spectroscopy is an effective new approach to the study of anharmonicity. The strength of the piezomodulation response for the modes most affected by the phase transition indicates that piezomodulated Raman spectroscopy can be a useful way of locating soft modes without the necessity of observing the spectra for all of the phases involved. In addition, the measurement of the coupling permits study of the anharmonicity. Frequency shifts in the elastic regime may be determined with an accuracy which may not be achievable by any other means. Determination of these properties by other methods can be onerous, because of the small size and fragility of the samples which can be destroyed under conditions required for the measurements.

Piezomodulated Raman spectroscopy is demonstrated to be an effective new probe for the study of lattice dynamics. The experiment is not a priori limited to molecular crystals, although their greater compressibilities ensure large responses. The ability to probe the anharmonicity of the lattice potential, as well as its anisotropy, also recommends piezomodulated Raman spectroscopy for the study of intermolecular forces.

One of us (K.M.W.) acknowledges receipt of a $\mathrm{Na}$ tional Science Foundation Graduate Fellowship.

\footnotetext{
(a) Author to whom correspondence should be addressed.

${ }^{1}$ T. Luty and C. J. Eckhardt, J. Chem. Phys. 82, 1515 (1985).

${ }^{2}$ C. L. Choy, S. P. Wong, and K. Young, Phys. Rev. B 29, 1741 (1984).

${ }^{3}$ R. W. Munn, Phys. Rev. B 12, 3491 (1975).
} 
${ }^{4}$ K. M. White, R. C. Dye, and C. J. Eckhardt, Mol. Cryst. Liq. Cryst. 134, 265 (1986).

${ }^{5}$ G. M. Parkinson, M. J. Goringe, S. Ramdas, J. O. Williams, and J. M. Thomas, J. Chem. Soc. Chem. Commun. 1978, 135.

${ }^{6}$ K. Schaum, K. Schaeling and F. Klausing, Liebigs Ann. Chem. 411, 161 (1916); F. B. Johnson, Nature (London) 178, 590 (1956).
${ }^{7}$ G. Gafner and F. H. Herbstein, Acta Crystallogr. 13, 706 (1960), and 17, 982 (1964).

${ }^{8}$ B. Pasquier and N. LeCalve, J. Raman Spectrosc. 6, 155 (1977).

${ }^{9}$ K. M. White and C. J. Eckhardt, to be published.

${ }^{10}$ E. Burgos and H. Bonadeo, Chem. Phys. Lett. 57, 125 (1978).

${ }^{11}$ S. D. Hamann, High Temp. High Pressure 8, 317 (1976). 